\title{
Efeitos sedativo e cardiorrespiratório da administração da metadona, isoladamente ou em associação à acepromazina ou xilazina, em gatos
}

Eduardo Raposo MONTEIRO ${ }^{1}$

Fabricia Mateus PICOLI ${ }^{1}$ Marisa Guizeline de Oliveira QUEIROZ1

Daniela CAMPAGNOL ${ }^{2}$

Juliany Gomes QUITZAN ${ }^{1}$

\section{Correspondência para:}

Eduardo Raposo Monteiro-Rua Alexandra, 215, Itanhangá, Rio de Janeiro-RJ, 22641530; btraposo@hotmail.com

Recebido para publicação: 06/09/2007 Aprovado para publicação: 24/04/2008

1 - Centro Universitário de Maringá, Maringá-PR

2 - Programa de Pós-Graduação em Anestesiologia da Faculdade de Medicina da Universidade Estadual Paulista, Botucatu-SP

\section{Resumo}

Seis felinos com peso médio de $3,3 \pm 0,3 \mathrm{~kg}$ foram aleatoriamente submetidos a 6 tratamentos, com intervalo mínimo de 1 semana. Os animais receberam a administração intramuscular de solução fisiológica (controle), metadona $(0,3 \mathrm{mg} / \mathrm{kg})$, acepromazina $(0,1 \mathrm{mg} / \mathrm{kg})$, xilazina $(1,0 \mathrm{mg} / \mathrm{kg})$, acepromazina $(0,05 \mathrm{mg} / \mathrm{kg})+$ metadona $(0,3 \mathrm{mg} / \mathrm{kg})$ ou xilazina $(0,5 \mathrm{mg} / \mathrm{kg})+$ metadona $(0,3 \mathrm{mg} / \mathrm{kg})$. As freqüências cardíaca (FC) e respiratória (FR), a pressão arterial sistólica (PAS), a temperatura retal, o grau de sedação e o reflexo interdigital foram avaliados antes (basal) e após a administração dos tratamentos em intervalos específicos por 90 minutos. Nos animais tratados com xilazina ou xilazina/metadona, houve diminuição em FR, FC e na temperatura retal. Nos mesmos tratamentos, $1 / 6$ e 2/6 animais não apresentaram reflexo interdigital em pelo menos um dos momentos avaliados. Nos animais que receberam a administração de $0,1 \mathrm{mg} / \mathrm{kg}$ de acepromazina, houve diminuição em PAS. Os escores de sedação foram mais elevados nos animais que receberam a administração de xilazina ou xilazina associada à metadona. A administração da metadona isolada ou associada à acepromazina resultou em sedação considerada insatisfatória e sinais de excitação em alguns animais. $\mathrm{O}$ uso da metadona isolado ou em associação à acepromazina foi considerado ineficaz quando se objetiva sedação moderada à intensa. A associação da metadona à xilazina produz sedação moderada à intensa, sendo esse efeito semelhante àquele observado após a administração da xilazina isoladamente em dose mais elevada.

\section{Introdução}

Os fármacos pertencentes ao grupo dos fenotiazínicos são utilizados primariamente para produzir sedação em pacientes veterinários. ${ }^{1}$ Adicionalmente, a administração desses fármacos apresenta outras vantagens tais como efeitos antiemético e antiarritmogênico, indução e recuperação anestésicas mais suaves ${ }^{2} \mathrm{e}$ diminuição na concentração alveolar mínima (CAM) de anestésicos inalatórios ${ }^{3}$. Em gatos a administração de acepromazina em doses variando de 0,03 a $0,1 \mathrm{mg} / \mathrm{kg}$ resulta em grau de sedação variável, embora esse efeito raramente permita a contenção do animal. ${ }^{4}$
Sedação mais pronunciada pode ser conseguida com a administração de fármacos pertencentes à classe dos agonistas alfa-2. A administração da xilazina a gatos produz sedação e analgesia dosedependentes. ${ }^{4}$ Após injeção intramuscular de $1 \mathrm{mg} / \mathrm{kg}$ de xilazina, observou-se sedação moderada ${ }^{5}$, embora sedação profunda possa ser conseguida quando doses mais elevadas são utilizadas ${ }^{4}$. No entanto, a administração de doses elevadas de fármacos agonistas alfa-2 resulta em acentuada depressão cardiovascular. ${ }^{6}$

A metadona é um agente da classe dos opióides com efeitos farmacológicos similares aos da morfina. Esse opioíde atua 
em receptores opióides do tipo mu, sendo esse o provável mecanismo pelo qual esse fármaco provoca seus efeitos analgésicos. ${ }^{7}$ Acredita-se ainda, que a metadona possa atuar como antagonista de receptores NMDA na medula espinhal, característica essa que pode incrementar a analgesia proporcionada por esse fármaco e diminuir a ocorrência de tolerância pelo seu uso contínuo. $^{8}$

Associações de fármacos com propriedades sedativa e analgésica são freqüentemente utilizadas na medicina veterinária, sendo esse tipo de associação denominado neuroleptoanalgesia. ${ }^{1}$ As principais vantagens do emprego dessas associações são a obtenção de maior grau de sedação e analgesia.

Embora o emprego da metadona associada a fármacos sedativos tenha sido relatado como de uso rotineiro na anestesia de gatos ${ }^{4}$, não há estudos na literatura consultada sobre os efeitos sedativo e analgésico, bem como os efeitos sobre as variáveis cardiovasculares e respiratórias, resultantes da administração desse opióide, administrado isoladamente ou em associação a fármacos tranqüilizantes/sedativos, nessa espécie.

O presente estudo objetivou avaliar o efeito sedativo e os efeitos cardiorrespiratórios da administração da metadona isoladamente ou em associação à acepromazina ou xilazina em gatos.

\section{Material e Método}

Seis felinos adultos de raças mestiças (três machos e três fêmeas) considerados hígidos e com peso médio de $3,3 \mathrm{~kg}(3,3 \pm 0,3$ [média \pm desvio padrão]) foram utilizados no estudo. A seleção dos animais foi baseada em exame físico. Todos os animais foram submetidos a jejum alimentar de 12 horas, não sendo, no entanto, privados de água. Os animais receberam a administração de seis tratamentos, de forma aleatória, com intervalo mínimo de uma semana. Todos os tratamentos foram administrados na musculatura posterior da coxa. No tratamento controle foi administrada solução fisiológica $(0,1 \mathrm{ml} / \mathrm{kg})$. Os demais tratamentos foram: metadona $0,3 \mathrm{mg} / \mathrm{kg}$ (MET); acepromazina $0,1 \mathrm{mg} / \mathrm{kg}$ (ACP); xilazina $1,0 \mathrm{mg} / \mathrm{kg}$ (XIL); acepromazina 0,05 $\mathrm{mg} / \mathrm{kg}+$ metadona $0,3 \mathrm{mg} / \mathrm{kg}$ (ACPMET); xilazina $0,5 \mathrm{mg} / \mathrm{kg}+$ metadona $0,3 \mathrm{mg} / \mathrm{kg}$ (XILMET). Em todos os tratamentos, o volume foi corrigido com solução fisiológica a fim de se atingir um volume final equivalente a $0,1 \mathrm{ml} / \mathrm{kg}$. Um único observador, o qual não sabia a qual dos tratamentos o animal havia sido submetido, foi o responsável por avaliar o grau de sedação e as variáveis cardiorrespiratórias. Todos os animais foram previamente aclimatados a uma sala com temperatura ambiente de aproximadamente $25{ }^{\circ} \mathrm{C}$ durante um período de 30 minutos. A seguir foram avaliados os valores basais de pressão arterial sistólica (PAS), freqüências cardíaca (FC) e respiratória (FR) e temperatura retal. A pressão arterial sistólica foi mensurada por meio de um doppler ultra-sônico, cujo sensor foi posicionado sobre a artéria digital palmar e um manguito de pressão, com largura de aproximadamente $40-50 \%$ da circunferência do membro, foi posicionado acima da articulação do carpo. Para a mensuração de FC utilizou-se o som da pulsação amplificado pelo doppler ultrassônico. Bradicardia e hipotensão foram consideradas freqüência cardíaca inferir a 100 batimentos por minuto e pressão arterial sistólica inferior a $104 \mathrm{mmHg}$, respectivamente. A freqüência respiratória foi mensurada por visualização dos movimentos torácicos e a temperatura retal, por meio de um termômetro clínico digital. O escore de sedação foi avaliado de acordo com a seguinte escala: 0 - sedação ausente; 1 - sedação leve (alerta porém menos ativo); 2 - sedação moderada (animal sonolento e em decúbito, porém capaz de andar); e 3- sedação intensa (animal muito sonolento e incapaz de andar). O reflexo interdigital foi avaliado pelo pinçamento da prega interdigital do membro torácico com uma pinça hemostática até a terceira cremalheira. Todas as variáveis, com exceção da 
temperatura retal, foram mensuradas antes (basal) e aos 5, 15, 30, 45, 60, 75 e 90 minutos após a administração dos tratamentos. A temperatura retal foi mensurada no momento basal e aos 90 minutos.

Para a análise estatística das variáveis paramétricas utilizou-se a análise de variância (ANOVA) seguida pelo teste de Tukey, Correção de Bonferroni ou teste de Dunnet, conforme apropriado. Diferenças entre os tratamentos e dentro de cada tratamento no escore de sedação foram comparadas por meio dos testes de Friedman e teste de Dunn. Todos os testes foram realizados ao nível de $5 \%$ de significância.

\section{Resultados}

Não houve diferença entre os tratamentos nos valores basais de FR, FC, PAS e temperatura. Os animais que receberam a administração de xilazina (XIL e XILMET) apresentaram os valores mais baixos de FC. A diferença foi significativa em relação aos demais tratamentos dos 15 aos 90 minutos após a administração dos tratamentos. Aos 5 minutos, FC foi mais baixa em XILMET do que em MET, ACP e ACPMET e mais baixa em XIL do que em MET e ACP. Dos 5 aos 90 minutos, FC foi significativamente mais baixa em relação aos valores basais em XIL e XILMET. Nos demais tratamentos, os valores de FC não diferiram significativamente ao longo do tempo (Tabela 1). Bradicardia (FC $<100$ bpm) foi observada em 5/6 e 4/6 animais dos tratamentos XIL e XILMET, respectivamente.

Os valores de PAS foram inferiores em ACP em relação a XIL aos 5 e 30 minutos. Não foram observadas outras diferenças entre os tratamentos nos valores de PAS, porém foi significativamente inferior no tratamento ACP dos 5 aos 90 minutos em relação ao valor basal (Tabela 1$)$.

Os animais que receberam a administração de xilazina (XIL e XILMET) apresentaram os valores médios mais baixos de FR. Apesar de terem ocorrido diferenças entre tratamentos em alguns momentos, os valores de FR se mantiveram dentro de valores considerados fisiológicos para o gato durante todo o estudo em todos os tratamentos (Tabela 1). Não foram observados episódios de apnéia ou cianose em nenhum dos tratamentos.

Os animais que receberam a administração de xilazina apresentaram os valores mais elevados no escore de sedação. Quatro dos seis animais nos tratamentos XIL e XILMET apresentaram sedação intensa (escore de sedação $=3$ ) em pelo menos um momento durante o estudo. Os animais restantes $(2 / 6)$ apresentaram sedação moderada (escore $=2)$. No tratamento controle, todos os animais apresentaram escore de sedação igual a zero (ausência de sedação) durante todo o estudo. Nos tratamentos MET e ACPMET, o grau de sedação observado variou de ausente $(4 / 6$ animais) à sedação leve (escore $=1 ; 2 / 6$ animais). No tratamento ACP, 4/6 animais apresentaram ausência de sedação, $1 / 6$ animal apresentou sedação leve e $1 / 6$ animal apresentou sedação moderada. O escore de sedação foi significativamente mais elevado em XIL e XILMET em relação ao tratamento controle aos 30 e 45 minutos. Quando comparado aos valores basais, o escore de sedação foi mais elevado em XIL e XILMET dos 30 aos 75 minutos após a administração dos tratamentos (Tabela 2).

Aos 90 minutos após a administração dos tratamentos, os valores médios de temperatura foram inferiores em XIL $(37,0 \pm 1,1)$ em relação ao controle $(38,3 \pm 0,3)$, MET $(38,5 \pm 1,0)$ e ACPMET $(38,3 \pm 0,3)$. Adicionalmente, a temperatura retal diminuiu em relação aos valores basais em XIL e em XILMET aos 90 minutos. A redução média na temperatura foi de $1,0^{\circ} \mathrm{C}$ e $0,8^{\circ} \mathrm{C}$ em XIL e XILMET, respectivamente (Tabela 3).

$\mathrm{O}$ reflexo interdigital foi ausente em $1 / 6$ animal no tratamento XIL e em 2/6 animais do tratamento XILMET. No animal submetido à administração isolada de xilazina a perda do reflexo ocorreu somente aos 45 minutos. No tratamento XILMET, em 1 animal o reflexo interdigital esteve ausente dos 5 aos 90 minutos, enquanto em outro 
Tabela 1 - Valores médios \pm desvio padrão de freqüência respiratória (FR), freqüência cardíaca (FC) e pressão arterial sistólica (PAS) em 6 gatos antes (basal) e após a administração de salina (controle), metadona (MET), acepromazina (ACP), xilazina (XIL), acepromazina + metadona (ACPMET) ou xilazina + metadona (XILMET)

\begin{tabular}{|c|c|c|c|c|c|c|c|c|}
\hline \multirow[b]{2}{*}{ Tratamento } & \multirow[b]{2}{*}{ Basal } & \multicolumn{7}{|c|}{ Tempo (min) após a administração dos tratamentos } \\
\hline & & 5 & 15 & 30 & 45 & 60 & 75 & 90 \\
\hline \multicolumn{9}{|c|}{ FR (mpm) } \\
\hline Controle & $69 \pm 20^{\mathrm{a}}$ & $73 \pm 21^{\mathrm{a}}$ & $70 \pm 16^{a}$ & $71 \pm 22^{\mathrm{a}}$ & $56 \pm 23^{\mathrm{ab}}$ & $62 \pm 30^{\mathrm{ab}}$ & $61 \pm 27^{\mathrm{ab}}$ & $73 \pm 23^{\mathrm{a}}$ \\
\hline MET & $69 \pm 18^{a}$ & $67 \pm 19^{\mathrm{ab}}$ & $58 \pm 17^{\mathrm{ab}}$ & $63 \pm 20^{\mathrm{ab}}$ & $56 \pm 15^{a b}$ & $66 \pm 17^{2}$ & $69 \pm 10^{a}$ & $58 \pm 19^{\mathrm{ab}}$ \\
\hline $\mathrm{ACP}$ & $70 \pm 15^{\mathrm{a}}$ & $61 \pm 21^{\mathrm{ab}}$ & $61 \pm 27^{\mathrm{ab}}$ & $70 \pm 23^{\mathrm{a}}$ & $67 \pm 20^{\mathrm{a}}$ & $77 \pm 16^{a}$ & $69 \pm 12^{\mathrm{a}}$ & $60 \pm 16^{\mathrm{ab}}$ \\
\hline XIL & $57 \pm 21^{\mathrm{a}}$ & $50 \pm 13^{\mathrm{ab}}$ & $49 \pm 11^{\mathrm{ab}}$ & $45 \pm 8^{\mathrm{bc}}$ & $43 \pm 11^{\mathrm{b}}$ & $42 \pm 12^{\mathrm{bc}^{*}}$ & $41 \pm 10^{\mathrm{bc}}{ }^{*}$ & $39 \pm 11^{b c^{*}}$ \\
\hline ACPMET & $64 \pm 19^{a}$ & $53 \pm 21^{\mathrm{ab}}$ & $54 \pm 19^{\mathrm{ab}}$ & $53 \pm 27 \mathrm{abc}$ & $52 \pm 20^{\mathrm{ab}}$ & $62 \pm 33^{\mathrm{ab}}$ & $57 \pm 22^{\mathrm{ab}}$ & $61 \pm 25^{\mathrm{ab}}$ \\
\hline XILMET & $57 \pm 18^{2}$ & $49 \pm 22^{b}$ & $43 \pm 7 b$ & $39 \pm 7 \mathrm{c}^{*}$ & $35 \pm 5^{\mathrm{b}^{*}}$ & $34 \pm 10^{\mathrm{c}^{*}}$ & $31 \pm 4^{c^{*}}$ & $33 \pm 9 c^{*}$ \\
\hline \multicolumn{9}{|c|}{ FC (bpm) } \\
\hline Controle & $153 \pm 22^{\mathrm{a}}$ & $162 \pm 16^{\mathrm{abc}}$ & $164 \pm 14^{a}$ & $160 \pm 24^{a}$ & $156 \pm 17^{a}$ & $160 \pm 17^{\mathrm{a}}$ & $151 \pm 14^{\mathrm{a}}$ & $153 \pm 16^{a}$ \\
\hline MET & $172 \pm 30^{\mathrm{a}}$ & $179 \pm 28^{\mathrm{a}}$ & $167 \pm 31^{\mathrm{a}}$ & $163 \pm 33^{a}$ & $187 \pm 57^{a}$ & $178 \pm 40^{\mathrm{a}}$ & $173 \pm 39^{a}$ & $156 \pm 24^{a}$ \\
\hline $\mathrm{ACP}$ & $182 \pm 27^{a}$ & $194 \pm 29^{a}$ & $195 \pm 37^{a}$ & $175 \pm 43^{a}$ & $188 \pm 44^{a}$ & $172 \pm 48^{a}$ & $177 \pm 47^{a}$ & $174 \pm 43^{a}$ \\
\hline XIL & $182 \pm 35^{a}$ & $139 \pm 53^{\mathrm{bc}} \mathrm{c}^{*}$ & $120 \pm 45^{b^{*}}$ & $98 \pm 19^{b^{*}}$ & $91 \pm 16^{b^{*}}$ & $89 \pm 18^{b^{*}}$ & $91 \pm 13^{\mathrm{b}^{*}}$ & $98 \pm 28^{b^{*}}$ \\
\hline ACPMET & $163 \pm 24^{a}$ & $179 \pm 34^{\mathrm{ab}}$ & $185 \pm 33^{\mathrm{a}}$ & $163 \pm 20^{a}$ & $177 \pm 20^{\mathrm{a}}$ & $174 \pm 30^{\mathrm{a}}$ & $178 \pm 31^{\mathrm{a}}$ & $177 \pm 26^{a}$ \\
\hline XILMET & $167 \pm 40^{\mathrm{a}}$ & $123 \pm 25^{\mathrm{c}^{*}}$ & $123 \pm 49^{\mathrm{b}^{*}}$ & $99 \pm 16^{b^{*}}$ & $97 \pm 23^{\mathrm{b}^{*}}$ & $89 \pm 16^{\mathrm{b} *}$ & $95 \pm 18^{\mathrm{b}^{*}}$ & $97 \pm 22^{b^{*}}$ \\
\hline \multicolumn{9}{|c|}{ PAS (mm Hg) } \\
\hline Controle & $113 \pm 16^{a}$ & $123 \pm 11^{\mathrm{ab}}$ & $119 \pm 24^{a}$ & $113 \pm 15^{\mathrm{ab}}$ & $113 \pm 22^{a}$ & $128 \pm 25^{a}$ & $117 \pm 14^{a}$ & $119 \pm 21^{a}$ \\
\hline MET & $127 \pm 16^{a}$ & $116 \pm 12^{\mathrm{ab}}$ & $112 \pm 17^{a}$ & $126 \pm 17^{a b}$ & $115 \pm 15^{a}$ & $120 \pm 17^{a}$ & $119 \pm 17^{a}$ & $118 \pm 21^{a}$ \\
\hline $\mathrm{ACP}$ & $133 \pm 18^{a}$ & $109 \pm 19^{a^{*}}$ & $101 \pm 19^{a^{*}}$ & $102 \pm 18^{\mathrm{a}^{*}}$ & $106 \pm 8^{a^{*}}$ & $112 \pm 14^{2^{*}}$ & $105 \pm 14^{\mathrm{a}^{*}}$ & $102 \pm 19^{a^{*}}$ \\
\hline XII & $125 \pm 13^{a}$ & $139 \pm 20^{b}$ & $130 \pm 16^{a}$ & $135 \pm 13^{\mathrm{b}}$ & $128 \pm 21^{2}$ & $124 \pm 16^{a}$ & $120 \pm 17^{a}$ & $108 \pm 18^{a}$ \\
\hline ACPMET & $119 \pm 31^{\mathrm{a}}$ & $111 \pm 22^{\mathrm{ab}}$ & $111 \pm 31^{a}$ & $115 \pm 24^{\mathrm{ab}}$ & $109 \pm 23^{a}$ & $114 \pm 17^{a}$ & $113 \pm 15^{a}$ & $115 \pm 28^{a}$ \\
\hline XILMET & $131 \pm 21^{\mathrm{a}}$ & $130 \pm 22^{\mathrm{ab}}$ & $127 \pm 29^{a}$ & $129 \pm 25^{\mathrm{ab}}$ & $117 \pm 18^{a}$ & $118 \pm 23^{a}$ & $108 \pm 15^{a}$ & $107 \pm 16^{a}$ \\
\hline
\end{tabular}

Dentro de cada coluna (momentos), valores médios acompanhados de uma ou mais letras (indices) em comum não diferem significativamente entre si (ANOVA / Correção de Bonferroni, $\mathrm{P}<0,05$ ); * diferença significativa em relação ao valor basal (Teste de Dunnet, $\mathrm{P}<0,05$ )

animal esse reflexo esteve ausente dos 30 aos 60 minutos.

Observou-se que $5 / 6$ animais dos tratamentos XIL e XILMET apresentaram vômito, enquanto nenhum animal nos demais tratamentos apresentou esse efeito.

\section{Discussão e Conclusões}

Os fenotiazínicos, reconhecidamente, causam redução da pressão arterial. ${ }^{1}$ Esse efeito ocorre devido ao bloqueio de receptores adrenérgicos periféricos do tipo $a_{1}$, o qual resulta em diminuição da resistência vascular periférica com conseqüente redução na pressão arterial. Adicionalmente, o bloqueio alfa-adrenérgico, mediado pelas fenotiazinas, foi relatado como sendo dosedependente, fato esse demonstrado pela necessidade de doses progressivamente maiores de fenilefrina para elevar a pressão arterial média de cães tratados com 
Tabela 2 - Medianas (limite superior - limite inferior) dos escores de sedação em 6 gatos após a administração de salina (controle), metadona (MET), acepromazina (ACP), xilazina (XIL), acepromazina + metadona (ACPMET) ou xilazina + metadona (XILMET)

\begin{tabular}{|c|c|c|c|c|c|c|c|}
\hline \multirow[b]{2}{*}{ Tratamento } & \multicolumn{7}{|c|}{ Tempo (min) após a administração dos tratamentos } \\
\hline & 5 & 15 & 30 & 45 & 60 & 75 & 90 \\
\hline Controle & $0(0-0)^{\mathbf{a}}$ & $0(0-0)^{2}$ & $0(0-0)^{\mathbf{a}}$ & $0(0-0)^{\mathbf{a}}$ & $0(0-0)^{\mathbf{a}}$ & $0(0-0)^{2}$ & $0(0-0)^{\mathbf{a}}$ \\
\hline MET & $0(0-1)^{a}$ & $0(0-1)^{\mathbf{a}}$ & $0(0-1)^{\mathrm{ab}}$ & $0(0-1)^{\mathrm{ab}}$ & $0(0-1)^{\mathbf{2}}$ & $0(0-0)^{2}$ & $0(0-0)^{\mathbf{a}}$ \\
\hline $\mathrm{ACP}$ & $0(0-1)^{\mathrm{a}}$ & $0(0-1)^{\mathrm{a}}$ & $0(0-1)^{\mathrm{ab}}$ & $0(0-2)^{\mathrm{ab}}$ & $0(0-2)^{\mathbf{a}}$ & $0(0-1)^{\mathrm{a}}$ & $0(0-1)^{\mathbf{a}}$ \\
\hline XII & $0,5(0-2)^{a}$ & $1,5(0-3)^{\mathrm{a}}$ & $2,5(1-3)^{b *}$ & $3(1-3)^{b *}$ & $2,5(2-3)^{a *}$ & $2(2-3)^{a *}$ & $2(1-2)^{a}$ \\
\hline ACPMET & $0(0-0)^{a}$ & $0(0-1)^{\mathrm{a}}$ & $0(0-1)^{\mathrm{ab}}$ & $0(0-1)^{\mathrm{ab}}$ & $0(0-0)^{\mathbf{a}}$ & $0(0-0)^{\mathrm{a}}$ & $0(0-0)^{a}$ \\
\hline XILMET & $1(0-3)^{a}$ & $2(0-3)^{2}$ & $2(1-3)^{b *}$ & $3(2-3)^{b *}$ & $2,5(2-3)^{2 *}$ & $2(2-3)^{a *}$ & $2(1-2)^{2}$ \\
\hline
\end{tabular}

Dentro de cada coluna (momentos), medianas acompanhadas de uma ou mais letras (indices) em comum não diferem significativamente entre si (Friedman / Teste de Dunn, $\mathrm{P}<0,05$ ); * diferença significativa em relação ao valor basal (Friedman / Teste de Dunn, $\mathrm{P}<0,05$ )

Tabela 3 - Valores médios +desvio padrão de temperatura retal em 6 gatos antes (basal) e 90 minutos após a administração de salina (controle), metadona (MET), acepromazina $(\mathrm{ACP})$, xilazina (XIL), acepromazina + metadona (ACPMET) ou xilazina + metadona (XILMET)

\begin{tabular}{ccc}
\hline Tratamento & Basal & 90 \\
\hline Controle & $38.4 \pm 0.3^{\mathrm{a}}$ & $38.3 \pm 0.3^{\mathrm{a}}$ \\
MET & $38.4 \pm 0.3^{\mathrm{a}}$ & $38.5 \pm 1.0^{\mathrm{a}}$ \\
ACE & $38.3 \pm 0.6^{\mathrm{a}}$ & $37.9 \pm 0.6^{\mathrm{ab}}$ \\
XIL & $38.0 \pm 0.8^{\mathrm{a}}$ & $37.0 \pm 1.1^{\mathrm{b}^{*}}$ \\
ACEMET & $38.2 \pm 0.4^{\mathrm{a}}$ & $38.3 \pm 0.3^{\mathrm{a}}$ \\
XILMET & $38.3 \pm 0.6^{\mathrm{a}}$ & $37.5 \pm 0.8^{\mathrm{ab}}$
\end{tabular}

Dentro de cada coluna (momentos), valores médios acompanhados de uma ou mais letras (índices) em comum não diferem significativamente entre si (ANOVA / teste de Tukey, $\mathrm{p}<0,05) ; *$ diferença significativa em relação ao valor basal (teste t pareado, $\mathrm{p}<0,05$ )

acepromazina nas doses de 0,$05 ; 0,125$ e $0,25 \mathrm{mg} / \mathrm{kg} .{ }^{9}$ Corroborando com esses resultados, no presente estudo, houve diminuição significativa da pressão arterial em relação ao valor basal nos gatos que receberam a administração de acepromazina na dose de $0,1 \mathrm{mg} / \mathrm{kg}$, mas não naqueles tratados com $0,05 \mathrm{mg} / \mathrm{kg}$ em associação à metadona, sugerindo bloqueio alfaadrenérgico dose-dependente pela acepromazina. Adicionalmente, hipotensão (PAS inferior a $104 \mathrm{~mm} \mathrm{Hg}$ ) foi observada nos animais tratados com $0,1 \mathrm{mg} / \mathrm{kg}$ de acepromazina, mas não naqueles que receberam a administração de $0,05 \mathrm{mg} / \mathrm{kg}$.

Os efeitos cardiovasculares da administração da xilazina são característicos. Após administração intravenosa de xilazina $(1,0 \mathrm{mg} / \mathrm{kg})$ a cães, ocorre diminuição da freqüência cardíaca, elevação transitória da pressão arterial (por 5 a 10 minutos) seguida de diminuição para valores similares ou sensivelmente mais baixos do que os valores basais ${ }^{10,11,12}$, elevação na resistência vascular sistêmica e diminuição no débito cardíaco ${ }^{10,12}$. A elevação transitória da pressão arterial parece ser mediada pela estimulação de receptores vasculares pós-sinápticos, resultando em vasoconstrição. Já a diminuição subseqüente nesse parâmetro ocorre por diminuição do tônus simpático, a qual ocorre devido ao efeito central causado pela xilazina e pela ativação de receptores pré-sinápticos periféricos no sistema nervoso autônomo simpático ${ }^{2}$. A administração de xilazina pela via intramuscular resulta em efeitos similares, porém a fase hipertensiva pode não ser evidenciada ${ }^{10}$, provavelmente devido à concentração plasmática mais baixa de xilazina quando administrada por essa via ${ }^{2}$. Em gatos, a administração intramuscular de xilazina $(1,0 \mathrm{mg} / \mathrm{kg})$ resultou em diminuição da freqüência cardíaca a partir dos 5 minutos após a administração sem, no entanto, causar alterações significativas na pressão arterial. ${ }^{5}$ No presente estudo, a administração intramuscular de xilazina $(1,0 \mathrm{mg} / \mathrm{kg})$ resultou em elevação não significativa da 
pressão arterial aos 5 minutos após a injeção, seguida de diminuição para valores similares ao valor basal. No entanto, esse fato não ocorreu nos gatos tratados com a dose mais baixa de xilazina associada à metadona, provavelmente devido a um efeito menos acentuado da xilazina sobre os receptores vasculares pós-sinápticos com o uso da dose mais baixa. ${ }^{2}$ Por outro lado, o uso da dose mais baixa de xilazina não resultou em efeito cronotrópico negativo menos pronunciado. Houve diminuição na freqüência cardíaca nos gatos tratados com ambas as doses de xilazina já aos 5 minutos após a administração dos tratamentos e bradicardia (FC $<100 \mathrm{bpm}$ ) foi evidenciada dos 30 aos 90 minutos. Esses resultados corroboram com um estudo realizado em cães ${ }^{13}$, no qual os autores afirmaram que o uso de doses de medetomidina suficientes para produzir sedação serão invariavelmente acompanhadas de efeitos cardiovasculares.

Nas doses recomendadas para uso clínico, a administração de xilazina não resulta em alterações respiratórias significativas. Redução intensa da freqüência respiratória não foi acompanhada de alterações significativas na $\mathrm{PaO}_{2}, \mathrm{PaCO}_{2}$ ou no $\mathrm{pH}$ após a administração da xilazina no cão. ${ }^{10} \mathrm{Em}$ gatos, a administração intramuscular da xilazina $(1,0 \mathrm{mg} / \mathrm{kg})$ não resultou em alteração significativa na freqüência respiratória ou na saturação de oxigênio na hemoglobina. ${ }^{5}$ Contraditoriamente, no presente estudo houve diminuição significativa da freqüência respiratória após a administração isolada da xilazina. Essa divergência se deve provavelmente ao fato de os animais do presente estudo apresentarem valores basais de freqüência respiratória mais elevados. Após a administração da xilazina, os valores médios de FR observados no presente estudo foram similares àqueles observados no estudo realizado por Selmi et al. ${ }^{5}$. Os opióides são conhecidos por sua capacidade de promover depressão respiratória dosedependente, especialmente os agonistas de receptores mu, como a morfina, metadona e fentanil. ${ }^{1} \mathrm{Um}$ estudo prévio demonstrou que a associação da xilazina à l-metadona resultou em acidose respiratória em cães. ${ }^{14}$ No presente estudo, quando a metadona foi associada à xilazina, houve diminuição na freqüência respiratória de maior magnitude em comparação ao uso isolado de xilazina. Os valores de FR se mantiveram dentro de valores considerados normais para o gato durante todo o estudo, não sendo observado nenhum caso de cianose. No entanto, não é possível afirmar ao certo, a partir dos resultados do presente estudo, que a associação da xilazina à metadona não resulta em depressão respiratória, tendo em vista que os valores de pressão parcial de dióxido de carbono arterial $\left(\mathrm{PaCO}_{2}\right)$ ou expirado $\left(\mathrm{EtCO}_{2}\right)$ não foram mensurados.

Diminuição da temperatura corpórea foi relatada após a administração de agonistas alfa-2, sendo nesse caso relacionada à diminuição na produção de calor, devido à reduzida atividade muscular, bem como pela interferência nos mecanismos termorreguladores. ${ }^{15}$ No presente estudo, a administração de ambas as doses de xilazina utilizadas resultou em diminuição significativa da temperatura corpórea.

O vômito tem sido descrito como um efeito adverso da administração da xilazina em cães e gatos. ${ }^{16} \mathrm{Em}$ um estudo anterior realizado em gatos ${ }^{5}$, a incidência de vômito após a administração da xilazina foi de $80 \%$. No presente estudo, a incidência de vômito foi de $83 \%$ nos tratamentos XIL e XILMET. O uso da dose mais baixa de xilazina não resultou em menor incidência de vômito, sugerindo que esse efeito possa ser desencadeado com doses baixas de xilazina.

A associação de fenotiazinas e agonistas alfa- 2 aos analgésicos opióides vem sendo utilizada por muitos anos na medicina veterinária. ${ }^{1}$ Essas associações resultam em sinergismo, permitindo a utilização de doses mais baixas de cada fármaco, sendo a analgesia e a sedação observadas freqüentemente superiores em comparação ao uso de cada agente isoladamente. ${ }^{1}$ Tendo em vista que as doses utilizadas dos tranqüilizantes e opióides são mais baixas, 
poderia se esperar que os efeitos adversos resultantes da administração de cada fármaco sejam atenuados. No entanto, embora a associação de fenotiazinas a opióides tenha se mostrado uma técnica segura, capaz de provocar boa sedação no cão $\mathrm{O}^{1,17}$, esses resultados não foram reproduzidos em gatos ${ }^{4}$. Tem sido relatado na literatura que gatos tratados com doses elevadas de opióides podem apresentar excitação. ${ }^{4}$ Por essa razão, as doses de opióides utilizadas em associação a tranqüilizantes como a acepromazina são mais baixas em relação àquelas utilizadas em cães. Como resultado, o incremento no grau de sedação produzido pela associação de opióides à acepromazina, em relação ao uso isolado da fenotiazina, é mínimo ${ }^{4}$. No presente estudo o grau de sedação obtido pela administração da acepromazina $(0,1 \mathrm{mg} / \mathrm{kg})$ variou de sedação não evidente à sedação moderada. Quando uma dose mais baixa de acepromazina $(0,05 \mathrm{mg} / \mathrm{kg})$ foi associada à metadona, o grau de sedação não foi incrementado. Adicionalmente, sinais de excitação caracterizados por aumento da atividade locomotora foram evidenciados em 4/6 animais tratados com a associação acepromazina/metadona contra apenas $1 / 6$ animais tratados somente com acepromazina. Esse fato sugere um possível efeito excitatório mediado pela metadona na dose empregada no presente estudo. Embora os efeitos da acepromazina sobre a pressão arterial tenham sido amenizados com o uso de dose mais baixa da fenotiazina, a associação não demonstrou ser viável quando se objetiva sedação no gato. Por outro lado, quando uma dose mais baixa de xilazina $(0,5 \mathrm{mg} / \mathrm{kg})$ foi associada à metadona $(0,3 \mathrm{mg} / \mathrm{kg})$, observou-se grau de sedação semelhante àquele produzido pela administração de uma dose mais elevada de xilazina $(1,0 \mathrm{mg} / \mathrm{kg})$, sugerindo uma possível potencialização pela metadona sobre o efeito sedativo mediado pela administração da xilazina.
Um estudo comparando doses equivalentes de xilazina na presença ou ausência do opióide seria necessário para comprovar essa hipótese.

A administração subcutânea da metadona $(0,2 \mathrm{mg} / \mathrm{kg})$ resultou em elevação do limiar aos estímulos térmico e pressórico em gatos. ${ }^{18} \mathrm{O}$ uso isolado da metadona no presente estudo não resultou em ausência de reflexo interdigital em nenhum animal. No entanto, a associação da xilazina à metadona resultou em um maior número de animais com reflexo interdigital ausente em relação ao uso isolado da xilazina ( $2 / 6$ versus $1 / 6)$, sendo a duração desse efeito mais prolongada quando comparada ao uso isolado da xilazina. Embora esses resultados possam sugerir um melhor grau de analgesia quando a xilazina é associada à metadona em relação ao uso isolado da xilazina, esses resultados devem ser interpretados com cuidado, uma vez que fármacos que influenciam o grau de sedação e as respostas motoras podem interferir na resposta a um estímulo nociceptivo. ${ }^{19}$ Portanto não é possível afirmar, a partir dos resultados do presente estudo, que a associação da metadona à xilazina resulta em melhor analgesia do que o uso isolado do agonista alfa-2.

Os resultados do presente estudo permitem concluir que o uso da metadona isoladamente ou associada à acepromazina, nas doses utilizadas, resulta em grau de sedação insatisfatório, podendo haver episódios de excitação em ambos os casos. Quando se objetiva sedação moderada à intensa, a administração da xilazina isolada ou associada à metadona demonstrou ser eficaz. Todas as associações utilizadas foram bem toleradas por animais saudáveis. $\mathrm{O}$ principal efeito adverso foi a bradicardia, associada à administração de xilazina.

\section{Agradecimentos}

Ao PROBIC - Programa de Bolsas de Iniciação Científica - do Cesumar pela concessão de uma bolsa de iniciação científica. 


\title{
Sedative and cardiorespiratory effects of methadone, alone or in combination with acepromazine or xylazine, in cats
}

\begin{abstract}
Six cats weighting $3.3 \pm 0.3 \mathrm{~kg}$ were randomly allocated to 6 treatments, with at least one-week intervals. The cats received intramuscular administration of physiological saline (control), methadone $(0.3 \mathrm{mg} /$ $\mathrm{kg})$, acepromazine $(0,1 \mathrm{mg} / \mathrm{kg})$, xylazine $(1.0 \mathrm{mg} / \mathrm{kg})$, acepromazine $(0.05 \mathrm{mg} / \mathrm{kg})$ plus methadone $(0.3 \mathrm{mg} / \mathrm{kg})$ or xylazine $(0.5 \mathrm{mg} / \mathrm{kg})$ plus methadone $(0.3 \mathrm{mg} / \mathrm{kg})$. Heart rate $(\mathrm{HR})$, respiratory rate $(\mathrm{RR})$, indirect systolic arterial pressure (SAP), rectal temperature, sedation score and pedal withdrawal reflex were evaluated before (baseline) and at selected intervals after treatment administration for 90 minutes. Respiratory rate, HR and rectal temperature decreased in cats given xylazine or xylazine plus methadone. In 1 out of 6 cats given xylazine and 2 out of 6 cats given xylazine/methadone, pedal withdrawal reflex was absent. In cats given $0.1 \mathrm{mg} / \mathrm{kg}$ of acepromazine, SAP decreased compared to baseline. Sedation scores were greater in cats given xylazine or xylazine plus methadone. Methadone alone or in combination with acepromazine did not produce a satisfactory degree of sedation and resulted in signs of excitement in some of the cats. Methadone alone or combination with acepromazine was not considered an effective protocol when moderate to deep sedation is required in cats. Methadone in combination with xylazine produces moderate to deep sedation, being this effect comparable to that achieved with a higher dose of xylazine alone.
\end{abstract}

Key words: Methadone.

\section{Referências}

1 HALL, L. W.; CLARKE, K. W.; TRIM, C. M. Principles of sedation, analgesia and premedication. In: HALL, L. W.; CLARKE, K. W.; TRIM, C. M. Veterinary anaesthesia. 10. ed. London: WB Saunders, 2001. p. 75-112.

2 THURMON, J. C.; TRANQUILLI, W. J.; BENSON, G. J. Preanesthetics and anesthetic adjuncts. In: THURMON, J. C.; TRANQUILLI, W. J.; BENSON, G. J. Lumb \& Jones' veterinary anesthesia. 3. ed. Philadelphia: Lippincott Williams \& Wilkins, 1996. p. 183-209.

3 HEARD, D. J., WEBB, A. I.; DANIELS, R. T. Effect of acepromazine on the anesthetic requirement of halothane in the dog. American Journal of Veterinary Research, v. 47, n. 10, p. 2113-2115, 1986.

4 HALL, L. W.; CLARKE, K. W.; TRIM, C. M. Anaesthesia of the cat. In: HALL, L. W.; CLARKE, K. W.; TRIM, C. M. Veterinary anaesthesia. 10. ed. London: WB Saunders, 2001. p. 441-461.

5 SELMI, A. L. et al. Sedative, analgesic and cardiorespiratory effects of romifidine in cats. Veterinary Anaesthesia and Analgesia, v. 31, n. 3, p. 195-206, 2004.

6 DUNKLE, N. et al. Cardiac performance in cats after administration of xylazine or xylazine and glycopyrrolate: echocardiographic evaluations. American Journal of Veterinary Research, v. 47, n. 10, p. 2212-2216, 1986.

7 GUTSTEIN, H. B.; AKIL, H. Opioid analgesics. In: HARDMAN, J. G.; LIMBIRD, L. E. Goodman \& gilman's: the pharmacological basis of therapeutics. New York: McGraw-Hill, 2001. cap.23, p. 569-620.

8 WAGNER, A. E. Opioids. In: GAYNOR, J. S.; MUIR, W. W. Veterinary pain management. St. Louis: Mosby, 2002. p. 164-183.

9 LUDDERS, J. W. et al. Blood pressure response to phenylephrine infusion in halothane-anesthetized dogs given acetylpromazine maleate. American Journal of Veterinary Research, v. 44, n. 6, p. 996-999, 1983.

10 KLIDE, A. M.; CALDERWOOD, H. W.; SOMA, L. R. Cardiopulmonary effects of xylazine in dogs. American Journal of Veterinary Research, v. 36, n. 7, p. 931-935, 1975.

$11 \mathrm{HSU}$, W. H.; LU, Z. X.; HEMBROUGH, F. B. Effect of xylazine on heart rate and arterial blood pressure in conscious dogs, as influenced by atropine, 4aminopyridine, doxapram, and yohimbine. Journal of the American Veterinary Medical Association, v. 186, n. 2, p. 153-156, 1985.

12 HASKINS, S. C.; PATZ, J. D.; FARVER, T. B. Xylazine 
and xylazine-ketamine in dogs. American Journal of Veterinary Research, v. 47, n. 3, p. 636-641, 1986.

13 PYPENDOP, B. H.; VERSTEGEN, J. P. Hemodynamic effects of medetomidine in the dog: a dose titration study. Veterinary Surgery, v. 27, n. 6, p. 612-622, 1998.

14 KRAMER, S.; NOLTE, I.; JOCHLE, W. Clinical comparison of medetomidine with xylazine/lmethadone in dogs. Veterinary Record, v. 138, n. 6, p. 128-133, 1996.

15 VIRTANEN, R. Pharmacological profiles of medetomidine and its antagonist, atipamezole. Acta Veterinaria Scandinavica Supplementum, v. 85, p. 2937, 1989.

16 LEMKE, K. A. Perioperative use of selective alpha-2 agonists and antagonists in small animals. Canadian Veterinary Journal, v. 45, n. 6, p. 475-480, 2004.

17 STEPIEN, R. L. et al. Cardiorespiratory effects of acepromazine maleate and buprenorphine hydrochloride in clinically normal dogs. American Journal of Veterinary Research, v. 56, n. 1, p. 78-84, 1995.

18 STEAGALL, P. V. et al. Effects of subcutaneous methadone, morphine, buprenorphine or saline on thermal and pressure thresholds in cats. Journal of Veterinary Pharmacology and Therapeutics, v. 29, n. 6, p. 531-537, 2006.

19 ANSAH, O. B.; RAEKALLIO, M.; VAINIO, O. Comparison of three doses of dexmedetomidine with medetomidine in cats following intramuscular administration. Journal of Veterinary Pharmacology and Therapeutics, v. 21, n. 5, p. 380-387, 1998. 